\title{
A Note on a Beam Equation with Nonlinear Boundary Conditions
}

\author{
Paolamaria Pietramala \\ Dipartimento di Matematica, Università della Calabria, Arcavacata di Rende 87036, Cosenza, Italy \\ Correspondence should be addressed to Paolamaria Pietramala, pietramala@unical.it
}

Received 14 May 2010; Revised 12 July 2010; Accepted 31 July 2010

Academic Editor: Feliz Manuel Minhós

Copyright (c) 2011 Paolamaria Pietramala. This is an open access article distributed under the Creative Commons Attribution License, which permits unrestricted use, distribution, and reproduction in any medium, provided the original work is properly cited.

We present new results on the existence of multiple positive solutions of a fourth-order differential equation subject to nonlocal and nonlinear boundary conditions that models a particular stationary state of an elastic beam with nonlinear controllers. Our results are based on classical fixed point index theory. We improve and complement previous results in the literature. This is illustrated in some examples.

\section{Introduction}

The fourth-order differential equation

$$
u^{(4)}(t)=g(t) f(t, u(t)), \quad t \in(0,1)
$$

arises naturally in the study of the displacement $u=u(t)$ of an elastic beam when we suppose that, along its length, a load is added to cause deformations. This classical problem has been widely studied under a variety of boundary conditions (BCs) that describe the controls at the ends of the beam. In particular, Gupta [1] studied, along other sets of local homogeneous $\mathrm{BCs}$, the problem

$$
u(0)=0, \quad u^{\prime \prime}(0)=0, \quad u^{\prime}(1)=0, \quad u^{\prime \prime \prime}(1)=0
$$

that models a bar with the left end being simply supported (hinged) and the right end being sliding clamped. This problem, and its generalizations, has been studied previously by Davies and coauthors [2], Graef and Henderson [3] and Yao [4]. 
Multipoint versions of this problem do have a physical interpretation. For example, the four-point boundary conditions

$$
u(0)=0, \quad u^{\prime \prime}(0)=0, \quad u^{\prime}(1)=H_{1}(u(\tau)), \quad u^{\prime \prime \prime}(1)+H_{2}(u(\xi))=0
$$

model a bar where the displacement $u$ and the bending moment $u^{\prime \prime}$ at $t=0$ are zero, and there are relations, not necessarily linear, between the shearing force $u^{\prime \prime \prime}$ and the angular attitude $u^{\prime}$ at $t=1$ and the displacement $u$ in two other points of the beam.

In this paper we establish new results on the existence of positive solutions for the fourth-order differential equation (1.1) subject to the following nonlocal nonlinear boundary conditions:

$$
\begin{gathered}
u(0)=0, \quad u^{\prime \prime}(0)=0, \\
u^{\prime}(1)=H_{1}\left(\alpha_{1}[u]\right), \\
u^{\prime \prime \prime}(1)+H_{2}\left(\alpha_{2}[u]\right)=0,
\end{gathered}
$$

where $H_{1}, H_{2}$ are nonnegative continuous functions and $\alpha_{1}[\cdot], \alpha_{2}[\cdot]$ are linear functionals given by

$$
\alpha_{1}[u]=\int_{0}^{1} u(s) d A_{1}(s), \quad \alpha_{2}[u]=\int_{0}^{1} u(s) d A_{2}(s),
$$

involving Riemann-Stieltjes integrals.

The conditions (1.5)-(1.6) cover a variety of cases and include, as special cases when $H_{1}(w)=H_{2}(w)=w$, multipoint and integral boundary conditions. These are widely studied objects in the case of fourth-order BVPs; see, for example, [5-14]. BCs of nonlinear type also have been studied before in the case of fourth-order equations; see, for example, [15-20] and references therein.

The study of positive solutions of BVPs that involve Stieltjes integrals has been done, in the case of positive measures, in [21-24]. Signed measures were used in [12, 25]; here, as in $[21,22]$, due to some inequalities involved in our theory, the functionals $\alpha_{i}[\cdot]$ are assumed to be given by positive measures.

A standard methodology to solve (1.1) subject to local BCs is to find the corresponding Green's function $k$ and to rewrite the BVP as a Hammerstein integral equation of the form

$$
u(t)=\int_{0}^{1} k(t, s) g(s) f(s, u(s)) d s
$$

However, for nonlocal and nonlinear BCs the form of Green's functions can become very complicated. In the case of linear, nonlocal BCs, an elegant approach is due to Webb and 
Infante [12], where a unified method is given to study a large class of problems. This is done via an auxiliary perturbed Hammerstein integral equation of the form

$$
u(t)=\gamma_{1}(t) \alpha_{1}[u]+\gamma_{2}(t) \alpha_{2}[u]+\int_{0}^{1} k(t, s) g(t) f(s, u(s)) d s,
$$

with suitable functions $\gamma_{1}, \gamma_{2}$.

Infante studied in $[26,27]$ the case of one nonlinear BC and in [21] a thermostat model with two nonlinear controllers. The approach used in [21] relied on an extension of the results of [25], valid for equations of the type

$$
u(t)=\gamma_{1}(t) H_{1}\left(\alpha_{1}[u]\right)+\gamma_{2}(t) H_{2}\left(\alpha_{2}[u]\right)+\int_{0}^{1} k(t, s) g(s) f(s, u(s)) d s,
$$

and gives a simple general method to avoid long technical calculations.

In our paper the approach of [21] is applied to BVP (1.1)-(1.6): we rewrite this BVP as a perturbed Hammerstein integral equation, and we prove the existence of multiple positive solutions under a suitable oscillatory behavior of the nonlinearity $f$. We observe that our results are new even for the local BCs, when $H_{1}\left(\alpha_{1}[u]\right)=H_{2}\left(\alpha_{2}[u]\right)=0$. We illustrate our theory with some examples. We also point out that this approach may be utilized for other sets of nonlinear BCs that have a physical interpretation, this is done in the last section.

\section{The Boundary Value Problem}

We begin by considering the homogeneous BVP

$$
u^{(4)}(t)=g(t) f(t, u(t)), \quad u(0)=u^{\prime \prime}(0)=u^{\prime}(1)=u^{\prime \prime \prime}(1)=0, \quad t \in(0,1),
$$

of which we seek an equivalent integral formulation of the form

$$
u(t)=\int_{0}^{1} k(t, s) g(s) f(s, u(s)) d s
$$

Due to the nature of these particular BCs, the Green's function $k$ can be constructed (as in [4]) by means of an auxiliary second-order BVP, namely,

$$
u^{\prime \prime}(t)+g(t) f(t, u(t))=0, \quad u(0)=0, \quad u^{\prime}(1)=0, \quad t \in(0,1)
$$

The solutions of the BVP (2.3) can be written in the form

$$
u(t)=\int_{0}^{1} G(t, s) g(s) f(s, u(s)) d s,
$$


where

$$
G(t, s)= \begin{cases}s, & s \leq t \\ t, & s>t .\end{cases}
$$

Therefore the function $k$ in $(2.2)$ is given by

$$
k(t, s)=\int_{0}^{1} G(t, v) G(v, s) d v
$$

In order to use the approach of $[21,25,28]$, we need to use some monotonicity properties of $k$. Now, since

$$
\begin{aligned}
G(t, v) G(v, s)= & v^{2} X_{[0, t]}(v) X_{[0, s]}(v)+v s X_{[0, t]}(v) X_{[s, 1]}(v) \\
& +t v X_{[t, 1]}(v) X_{[0, s]}(v)+t s X_{[t, 1]}(v) X_{[s, 1]}(v)
\end{aligned}
$$

we obtain the following formulation for the Green's function:

$$
k(t, s)= \begin{cases}\frac{s}{6}\left(-s^{2}-3 t^{2}+6 t\right), & s \leq t \\ \frac{t}{6}\left(-t^{2}-3 s^{2}+6 s\right), & s>t .\end{cases}
$$

We now look for a suitable interval $[a, b] \subset[0,1]$, a function $\Phi(s)$, and a constant $c_{\Phi}>0$ such that

$$
\begin{gathered}
k(t, s) \leq \Phi(s), \quad \text { for every }(t, s) \in[0,1] \times[0,1], \\
k(t, s) \geq c_{\Phi} \Phi(s), \quad \text { for every }(t, s) \in[a, b] \times[0,1] .
\end{gathered}
$$

Since $k$ is continuous on $[0,1] \times[0,1]$ and $k(t, s)>0$ for $(t, s) \in(0,1] \times(0,1]$, a natural choice could be

$$
\Phi(s)=\max _{(t, s) \in[0,1] \times[0,1]} k(t, s), \quad c_{\Phi}=\min _{(t, s) \in[a, b] \times[0,1]} \frac{k(t, s)}{\Phi(s)}
$$

here we look for a better $\Phi$, since this enables us to weaken the growth requirements on the nonlinearity $f$.

An upper bound for $k$ is obtained by finding $\max _{t \in[0,1]} k(t, s)$ for each fixed $s$. Since $(\partial / \partial t) k(t, s) \geq 0$ for $(t, s) \in[0,1] \times[0,1], k$ is a nondecreasing function of $t$ that attains its maximum, for each fixed $s$, when $t=1$.

Therefore, for $(t, s) \in[0,1] \times[0,1]$, we have

$$
k(t, s) \leq k(1, s)=\frac{s}{6}\left(-s^{2}+3\right):=\Phi(s) .
$$


Now, one can see that the derivative of the function $k(t, s) / \Phi(s)$ with respect to $s$ is non-positive for all $s \in[0,1]$, that is, the function $k(t, s) / \Phi(s)$ is a non-increasing function of $s$. Therefore, for an arbitrary $[a, b] \subset(0,1]$, we have

$$
k(t, s) \geq c_{\Phi} \Phi(s), \quad \text { for every }(t, s) \in[a, b] \times[0,1]
$$

where

$$
c_{\Phi}:=\min _{a \leq t \leq b} \frac{k(t, 1)}{\Phi(1)}=\frac{k(a, 1)}{\Phi(1)}=\frac{1}{2} a\left(3-a^{2}\right) .
$$

We now turn our attention to the BVP (1.1)-(1.6)

$$
u^{(4)}(t)=g(t) f(t, u(t)), \quad u(0)=u^{\prime \prime}(0)=u^{\prime}(1)-H_{1}\left(\alpha_{1}[u]\right)=u^{\prime \prime \prime}(1)+H_{2}\left(\alpha_{2}[u]\right)=0,
$$

and we show that we can study this problem by means of a perturbation of the Hammerstein integral equation (2.2).

In order to do this, we look for the (unique) solutions of the linear problems

$$
\begin{gathered}
\gamma_{1}{ }^{(4)}(t)=0, \quad \gamma_{1}(0)=\gamma_{1}^{\prime \prime}(0)=0, \quad \gamma_{1}^{\prime}(1)=1, \quad \gamma_{1}^{\prime \prime \prime}(1)=0, \\
\gamma_{2}{ }^{(4)}(t)=0, \quad \gamma_{2}(0)=\gamma_{2}^{\prime \prime}(0)=\gamma_{2}^{\prime}(1)=0, \quad \gamma_{2}^{\prime \prime \prime}(1)+1=0
\end{gathered}
$$

that are

$$
\gamma_{1}(t)=t, \quad \gamma_{2}(t)=-\frac{1}{6} t^{3}+\frac{1}{2} t
$$

We observe that, for an arbitrary $[a, b] \subset(0,1]$, we have

$$
\begin{gathered}
\left\|\gamma_{1}\right\|=1, \quad \min _{t \in[a, b]} \gamma_{1}(t)=\gamma_{1}(a)=a, \\
\left\|\gamma_{2}\right\|=\frac{1}{3}, \quad \min _{t \in[a, b]} \gamma_{2}(t)=\gamma_{2}(a)=-\frac{1}{6} a^{3}+\frac{1}{2} a,
\end{gathered}
$$

where $\|u\|:=\sup \{|u(t)|: t \in[0,1]\}$, and therefore

$$
\gamma_{1}(t) \geq c_{\gamma_{1}}\left\|\gamma_{1}\right\|, \quad \gamma_{2}(t) \geq c_{\gamma_{2}}\left\|\gamma_{2}\right\|, \quad \text { for every } t \in[a, b],
$$

with

$$
c_{\gamma_{1}}:=\frac{\min _{t \in[a, b]} \gamma_{1}(t)}{\left\|\gamma_{1}\right\|}=a, \quad c_{\gamma_{2}}:=\frac{\min _{t \in[a, b]} \gamma_{2}(t)}{\left\|\gamma_{2}\right\|}=-\frac{a^{3}}{2}+\frac{3 a}{2} .
$$



equation

By a solution of the BVP (1.1)-(1.6) we mean a solution of the perturbed integral

$$
u(t)=\gamma_{1}(t) H_{1}\left(\alpha_{1}[u]\right)+\gamma_{2}(t) H_{2}\left(\alpha_{2}[u]\right)+\int_{0}^{1} k(t, s) g(t) f(s, u(s)) d s=: T u(t),
$$

and we work in a suitable cone in the Banach space $C[0,1]$ of continuous functions defined on the interval $[0,1]$ endowed with the usual supremum norm.

Our assumptions are the following:

$\left(C_{1}\right) f:[0,1] \times[0, \infty) \rightarrow[0, \infty)$ satisfies Carathéodory conditions, that is, for each $u$, $t \mapsto f(t, u)$ is measurable, for almost every $t, u \mapsto f(t, u)$ is continuous, and for every $r>0$, there exists an $L^{\infty}$-function $\phi_{r}:[0,1] \rightarrow[0, \infty)$ such that

$$
f(t, u) \leq \phi_{r}(t) \text { for almost all } t \in[0,1] \text { and all } u \in[0, r]
$$

$\left(C_{2}\right) g \Phi \in L^{1}[0,1], g \geq 0$ almost everywhere, and $\int_{0}^{1} \Phi(s) g(s) d s>0$;

( $\left.C_{3}\right) H_{1}, H_{2}$ are positive continuous functions such that there exist $h_{11}, h_{12}, h_{21}, h_{22} \in$ $[0, \infty)$ with

$$
h_{11} v \leq H_{1}(v) \leq h_{12} v, \quad h_{21} v \leq H_{2}(v) \leq h_{22} v,
$$

for every $v \geq 0$;

( $\left.C_{4}\right) \alpha_{1}[\cdot], \alpha_{2}[\cdot]$ are positive bounded linear functionals on $C[0,1]$ given by

$$
\alpha_{i}[u]=\int_{0}^{1} u(s) d A_{i}(s), \quad i=1,2,
$$

involving Stieltjes integrals with positive measures $d A_{i}$;

$\left(C_{5}\right) D_{2}:=\left(1-h_{12} \alpha_{1}\left[\gamma_{1}\right]\right)\left(1-h_{22} \alpha_{2}\left[\gamma_{2}\right]\right)-h_{12} h_{22} \alpha_{1}\left[\gamma_{2}\right] \alpha_{2}\left[\gamma_{1}\right]>0, h_{12} \alpha_{1}\left[\gamma_{1}\right]<1$ and $h_{22} \alpha_{2}\left[\gamma_{2}\right]<1$.

It follows from this last hypothesis that

$$
D_{1}:=\left(1-h_{11} \alpha_{1}\left[\gamma_{1}\right]\right)\left(1-h_{21} \alpha_{2}\left[\gamma_{2}\right]\right)-h_{11} h_{21} \alpha_{1}\left[\gamma_{2}\right] \alpha_{2}\left[\gamma_{1}\right] \geq D_{2}>0 .
$$


The above hypotheses enable us to utilize the cone

$$
K=\left\{u \in C[0,1]: u \geq 0, \min _{t \in[a, b]} u(t) \geq c\|u\|\right\},
$$

for an arbitrary $[a, b] \subset(0,1]$ and

$$
c:=\min \left\{c_{\Phi}, c_{\gamma_{1}}, c_{\gamma_{2}}\right\}=a,
$$

and to use the classical fixed point index for compact maps (see e.g., [29] or [30]).

We observe, as in [21], that $T$ leaves $K$ invariant and is compact. We give the proof in the Carathéodory case for completeness.

Lemma 2.1. If the hypotheses $\left(C_{1}\right)-\left(C_{4}\right)$ hold, then $T$ maps $K$ into $K$. Moreover, $T$ is a compact map.

Proof. Take $u \in K$ such that $\|u\| \leq r$. Then we have, for $t \in[0,1]$,

$$
\begin{aligned}
T u(t) & =\gamma_{1}(t) H_{1}\left(\alpha_{1}[u]\right)+\gamma_{2}(t) H_{2}\left(\alpha_{2}[u]\right)+\int_{0}^{1} k(t, s) g(s) f(s, u(s)) d s \\
& \leq \gamma_{1}(t) H_{1}\left(\alpha_{1}[u]\right)+\gamma_{2}(t) H_{2}\left(\alpha_{2}[u]\right)+\int_{0}^{1} \Phi(s) g(s) \phi_{r}(s) d s,
\end{aligned}
$$

therefore

$$
\|T u\| \leq\left\|\gamma_{1}\right\| H_{1}\left(\alpha_{1}[u]\right)+\left\|\gamma_{2}\right\| H_{2}\left(\alpha_{2}[u]\right)+\int_{0}^{1} \Phi(s) g(s) \phi_{r}(s) d s
$$

Then we obtain

$$
\min _{t \in[a, b]} T u(t) \geq c_{\gamma_{1}}\left\|\gamma_{1}\right\| H_{1}\left(\alpha_{1}[u]\right)+c_{\gamma_{2}}\left\|\gamma_{2}\right\| H_{2}\left(\alpha_{2}[u]\right)+c_{\Phi} \int_{0}^{1} \Phi(s) g(s) \phi_{r}(s) \geq c\|T u\| .
$$

Hence we have $T u \in K$. Moreover, the map $T$ is compact since it is a sum of two compact maps: the compactness of $\int_{0}^{1} k(t, s) g(s) f(s, u(s)) d s$ is well known, and since $\gamma_{1}, \gamma_{2}, H_{1}$, and $H_{2}$ are continuous, the perturbation $\gamma_{1}(t) H_{1}\left(\alpha_{1}[u]\right)+\gamma_{2}(t) H_{2}\left(\alpha_{2}[u]\right)$ maps bounded sets into bounded subsets of a 1-dimensional space.

For $\rho>0$, we use, as in $[23,31]$, the following bounded open subsets of $K$ :

$$
K_{\rho}=\{u \in K:\|u\|<\rho\}, \quad V_{\rho}=\left\{u \in K: \min _{t \in[a, b]} u(t)<\rho\right\} .
$$

Note that $K_{\rho} \subset V_{\rho} \subset K_{\rho / c}$. 
We employ the following numbers:

$$
\begin{gathered}
f^{0, \rho}:=\sup _{0 \leq u \leq \rho, 0 \leq t \leq 1} \frac{f(t, u)}{\rho}, \quad f_{\rho, \rho / c}:=\inf _{\rho \leq u \leq \rho / c,} \frac{f(t, u)}{a \leq t \leq b} \frac{1}{\rho}, \\
\frac{1}{m}:=\sup _{t \in[0,1]} \int_{0}^{1} k(t, s) g(s) d s, \quad \frac{1}{M(a, b)}:=\inf _{t \in[a, b]} \int_{a}^{b} k(t, s) g(s) d s,
\end{gathered}
$$

and we note

$$
\mathcal{K}_{i}(s):=\int_{0}^{1} k(t, s) d A_{i}(t), \quad i=1,2
$$

The proofs of the following results can be immediately deduced from the analogous results in [21], where the proofs involve a careful analysis of fixed point index and utilize order-preserving matrices. The only difference here is that we allow nonlinearity $f$ to be Carathéodory instead of continuous. The lines of proof are not effected and therefore the proofs are omitted.

Firstly we give conditions which imply that the fixed point index is 0 on the set $V_{\rho}$.

Lemma 2.2. Suppose that $\left(C_{1}\right)-\left(C_{5}\right)$ hold. Assume that there exist $\rho>0$ such that

$$
\begin{aligned}
f_{\rho, \rho / c} & \left(\left(\frac{c_{\gamma_{1}}\left\|\gamma_{1}\right\|}{D_{1}}\left(1-h_{21} \alpha_{2}\left[\gamma_{2}\right]\right)+\frac{c_{\gamma_{2}}\left\|\gamma_{2}\right\|}{D_{1}} h_{11} \alpha_{2}\left[\gamma_{1}\right]\right) \int_{0}^{1} \mathcal{K}_{1}(s) g(s) d s\right. \\
& \left.+\left(\frac{c_{\gamma_{1}}\left\|\gamma_{1}\right\|}{D_{1}} h_{21} \alpha_{1}\left[\gamma_{2}\right]+\frac{c_{\gamma_{2}}\left\|\gamma_{2}\right\|}{D_{1}}\left(1-h_{11} \alpha_{1}\left[\gamma_{1}\right]\right)\right) \int_{0}^{1} \mathcal{K}_{2}(s) g(s) d s+\frac{1}{M(a, b)}\right)>1 .
\end{aligned}
$$

Then the fixed point index, $i_{K}\left(T, V_{\rho}\right)$, is 0 .

Now, we give conditions which imply that the fixed point index is 1 on the set $K_{\rho}$.

Lemma 2.3. Suppose $\left(C_{1}\right)-\left(C_{5}\right)$ hold. Assume that there exists $\rho>0$ such that

$$
\begin{aligned}
f^{0, \rho}( & \left(\frac{\left\|\gamma_{1}\right\|}{D_{2}}\left(1-h_{22} \alpha_{2}\left[\gamma_{2}\right]\right)+\frac{\left\|\gamma_{2}\right\|}{D_{2}} h_{12} \alpha_{2}\left[\gamma_{1}\right]\right) \int_{0}^{1} \mathcal{K}_{1}(s) g(s) d s \\
& \left.+\left(\frac{\left\|\gamma_{1}\right\|}{D_{2}} h_{22} \alpha_{1}\left[\gamma_{2}\right]+\frac{\left\|\gamma_{2}\right\|}{D_{2}}\left(1-h_{12} \alpha_{1}\left[\gamma_{1}\right]\right)\right) \int_{0}^{1} \mathcal{K}_{2}(s) g(s) d s+\frac{1}{m}\right)<1 .
\end{aligned}
$$

Then $i_{K}\left(T, K_{\rho}\right)=1$.

The two lemmas above lead to the following result on existence of one or two positive solutions for the integral equation (2.20). Note that, if the nonlinearity $f$ has a suitable oscillatory behavior, it is possible to state, with the same arguments as in [23], a theorem on the existence of more than two positive solutions. 
Theorem 2.4. Suppose $\left(C_{1}\right)-\left(C_{5}\right)$ hold. Let $[a, b] \subset(0,1)$ and $c$ be as in (2.26). Then (2.20) has one positive solution in $K$ if either of the following conditions holds:

$\left(S_{1}\right)$ there exist $\rho_{1}, \rho_{2} \in(0, \infty)$ with $\rho_{1}<\rho_{2}$ such that (2.34) is satisfied for $\rho_{1}$ and (2.33) is satisfied for $\rho_{2}$;

$\left(S_{2}\right)$ there exist $\rho_{1}, \rho_{2} \in(0, \infty)$ with $\rho_{1}<c \rho_{2}$ such that (2.33) is satisfied for $\rho_{1}$ and $(2.34)$ is satisfied for $\rho_{2}$.

Equation (2.20) has at least two positive solutions in $K$ if one of the following conditions hold.

$\left(D_{1}\right)$ there exist $\rho_{1}, \rho_{2}, \rho_{3} \in(0, \infty)$, with $\rho_{1}<\rho_{2}<c \rho_{3}$, such that (2.34) is satisfied for $\rho_{1}$, (2.33) is satisfied for $\rho_{2}$, and (2.34) is satisfied for $\rho_{3}$;

$\left(D_{2}\right)$ there exist $\rho_{1}, \rho_{2}, \rho_{3} \in(0, \infty)$, with $\rho_{1}<c \rho_{2}$ and $\rho_{2}<\rho_{3}$, such that (2.33) is satisfied for $\rho_{1},(2.34)$ is satisfied for $\rho_{2}$, and (2.33) is satisfied for $\rho_{3}$.

\section{Optimal Constants and Examples}

Consider the differential equation

$$
u^{(4)}(t)=f(t, u(t)), \quad t \in(0,1),
$$

with the BCs (1.4)-(1.6).

The value $1 / m$ is given by direct calculation as follows:

$$
\frac{1}{m}=\sup _{t \in[0,1]} \int_{0}^{1} k(t, s) d s=\max _{t \in[0,1]} \frac{t}{24}\left(t^{3}-4 t^{2}+8\right)=\frac{5}{24}
$$

We seek the "optimal" $[a, b]$ for which $M(a, b)$ is a minimum. This type of problems has been tackled in the past, for example, in the second-order case for heat-flow problems in [32] and for beam equations (under different BCs) in $[9,12,13]$.

The kernel $k$ is a positive, nondecreasing function of $t$, thus

$$
\frac{1}{M(a, b)}=\min _{t \in[a, b]} \int_{a}^{b} k(t, s) d s=\int_{a}^{b} k(a, s) d s=\int_{a}^{b} \frac{a}{6}\left(-a^{2}-3 s^{2}+6 s\right) d s .
$$

Since $k$ is a nondecreasing function of $t$, we have

$$
\max _{0<a<b \leq 1}\left\{\frac{1}{M(a, b)}\right\}=\max _{0<a \leq 1} \int_{a}^{1} \frac{a}{6}\left(-a^{2}-3 s^{2}+6 s\right) d s=\max _{0<a \leq 1}\left\{\frac{a}{6}\left(2 a^{3}-4 a^{2}+2\right)\right\} .
$$

Such maximum is attained at $a=1 / 2$. Thus the "optimal" interval $[a, b]$, for which $M(a, b)$ is a minimum, is the interval $[1 / 2,1]$; this gives $M(1 / 2,1)=48 / 5$ and $c=1 / 2$.

Remark 3.1. From Theorem 2.4, it is possible to state results for the existence of several nonnegative solutions for the homogeneous BVP

$$
u^{(4)}(t)=f(t, u(t)), \quad u(0)=u^{\prime \prime}(0)=u^{\prime}(1)=u^{\prime \prime \prime}(1)=0, \quad t \in(0,1) .
$$


For example, with $[a, b]=[1 / 2,1]$ and $c=11 / 16$, the BVP (3.5) has at least two positive solutions in $K$ if there exist $\rho_{1}<\rho_{2}<c \rho_{3}$, such that $f^{0, \rho_{1}}<4.8, f_{\rho_{2}, \rho_{2} / c}>9.6$ and $f^{0, \rho_{3}}<4.8$.

These results are new and improve and complement the previous ones. Gupta [1] and Yao [4] studied the problem with more general nonlinearity but established existence results only. Davies and co-authors [2] and Graef and Henderson [3] obtain the existence of multiple positive solutions for a $2 n$ th-order differential equation subject to our boundary conditions in the case $n=2$. In [2] the choice $[a, b]=[1 / 4,3 / 4]$ gives the values $\eta=4$ and $\mu=16^{4}$ which replace our constants $m$ and $M(1 / 2,1)$ in the growth conditions of $f$. The growth conditions of the nonlinearity $f=f(u)$ in Theorem 3.1 in [3] cannot be compared with ours, but we do not require the restriction $f(0) \neq 0$.

The next examples illustrate the applicability of our results. Firstly we consider, as an illustrative example, the case of a nonlinear 4-point problem.

Example 3.2. Consider the differential equation

$$
u^{(4)}(t)=f(t, u(t)), \quad t \in(0,1),
$$

with the BCs

$$
u(0)=0, \quad u^{\prime \prime}(0)=0, \quad u^{\prime}(1)=H_{1}(u(\tau)), \quad u^{\prime \prime \prime}(1)+H_{2}(u(\xi))=0,
$$

where $\tau, \xi \in(0,1)$ and, as in [22], for $i=1,2$

$$
H_{i}(w)= \begin{cases}\frac{1}{4 i} w, & 0 \leq w \leq 1 \\ \frac{1}{8 i} w+\frac{1}{8 i}, & w \geq 1\end{cases}
$$

In this case we have $h_{11}=1 / 8, h_{12}=1 / 4, h_{21}=1 / 16, h_{22}=1 / 8$,

$$
\begin{aligned}
\alpha_{1}\left[\gamma_{1}\right]=\tau, \quad \alpha_{1}\left[\gamma_{2}\right]=-\frac{1}{6}\left(\tau^{3}-3 \tau\right), \quad \alpha_{2}\left[\gamma_{1}\right]=\xi, \quad \alpha_{2}\left[\gamma_{2}\right]=-\frac{1}{6}\left(\xi^{3}-3 \xi\right), \\
\\
\int_{0}^{1} \mathcal{K}_{1}(s) d s=\int_{0}^{1} k(\tau, s) d s=\frac{\tau}{24}\left(\tau^{3}-4 \tau^{2}+8\right), \\
\int_{0}^{1} \mathcal{K}_{2}(s) d s=\int_{0}^{1} k(\xi, s) d s=\frac{\xi}{24}\left(\xi^{3}-4 \xi^{2}+8\right) .
\end{aligned}
$$


We now fix $\tau=1 / 4, \xi=1 / 2$ and show that all the constants that appear in (2.33) and in (2.34) can be computed. This choice leads to

$$
\alpha_{1}\left[\gamma_{1}\right]=\frac{1}{4}, \quad \alpha_{1}\left[\gamma_{2}\right]=\frac{47}{384}, \quad \alpha_{2}\left[\gamma_{1}\right]=\frac{1}{2}, \quad \alpha_{2}\left[\gamma_{2}\right]=\frac{11}{48}, \quad D_{2}=\frac{45373}{49152}
$$

Moreover we have

$$
\int_{0}^{1} \mathcal{K}_{1}(s) d s=\frac{497}{6144}, \quad \int_{0}^{1} \mathcal{K}_{2}(s) d s=\frac{19}{128}
$$

and conditions (2.34) and (2.33) read $f^{0, \rho_{1}}<2.8587$ and $f_{\rho_{2}, \rho_{2} / c}>6.9470$. Since $\left(S_{1}\right)$ holds, from Theorem 2.4 it follows that this BVP has a nontrivial solution in $K$. A nonlinearity that easily verifies $\left(S_{1}\right)$, for example, is the function $f(t, u)=u^{3}$ for every $0<\rho_{1}<\sqrt{2.8587}$ and every $\rho_{2}>\sqrt{6.9470}$.

We now give an example with continuously distributed positive measures.

Example 3.3. Consider the differential equation

$$
u^{(4)}(t)=f(t, u(t)), \quad t \in(0,1),
$$

with the BCs

$$
u(0)=0, \quad u^{\prime \prime}(0)=0, \quad u^{\prime}(1)=H_{1}\left(\int_{0}^{1} \alpha_{1} u(s) d s\right), \quad u^{\prime \prime \prime}(1)+H_{2}\left(\int_{0}^{1} \alpha_{2} u(s) d s\right)=0,
$$

with $\alpha_{1}, \alpha_{2}>0$ and $H_{i}, i=1,2$, as in the previous example. In this case, we obtain

$$
\alpha_{1}\left[\gamma_{1}\right]=\frac{\alpha_{1}}{2}, \quad \alpha_{1}\left[\gamma_{2}\right]=\frac{5 \alpha_{1}}{24}, \quad \alpha_{2}\left[\gamma_{1}\right]=\frac{\alpha_{2}}{2}, \quad \alpha_{2}\left[\gamma_{2}\right]=\frac{5 \alpha_{2}}{24}
$$

and for $i=1,2$

$$
\begin{gathered}
\mathcal{K}_{i}(s)=\int_{0}^{1} k(t, s) d A_{i}(t)=\int_{0}^{1} \alpha_{i} k(t, s) d t=\alpha_{i}\left(\frac{1}{24} s^{4}-\frac{1}{6} s^{3}+\frac{1}{3} s\right), \\
\int_{0}^{1} \mathcal{K}_{i}(s) d s=\frac{2 \alpha_{i}}{15} .
\end{gathered}
$$


The Condition $\left(C_{5}\right)$ becomes

$$
\alpha_{1}<8, \quad \alpha_{2}<\frac{192}{5}, \quad 1-\frac{5}{192} \alpha_{2}-\frac{1}{8} \alpha_{1}>0 .
$$

We now fix $\alpha_{1}=4, \alpha_{2}=8$. This choice leads to

$$
\begin{gathered}
\alpha_{1}\left[\gamma_{1}\right]=2, \quad \alpha_{1}\left[\gamma_{2}\right]=\frac{5}{6}, \quad \alpha_{2}\left[\gamma_{1}\right]=4, \quad \alpha_{2}\left[r_{2}\right]=\frac{5}{3}, \quad D_{2}=\frac{7}{24}, \\
\int_{0}^{1} \mathcal{K}_{1}(s) d s=\frac{8}{15}, \quad \int_{0}^{1} \mathcal{K}_{2}(s) d s=\frac{16}{15}
\end{gathered}
$$

and conditions (2.34) and (2.33) read $f^{0, \rho_{1}}<0.30713$ and $f_{\rho_{2}, \rho_{2} / c}>1.1166$.

\section{Other Nonlinear BCs}

So far we have discussed in detail the case of the BCs (1.4)-(1.6), but the same approach may be applied to (1.1) subject to the nonlinear BCs

$$
u(0)=H_{1}\left(\alpha_{1}[u]\right), \quad u^{\prime \prime}(0)+H_{2}\left(\alpha_{2}[u]\right)=0, \quad u^{\prime}(1)=0, \quad u^{\prime \prime \prime}(1)=0,
$$

or

$$
u(0)=H_{1}\left(\alpha_{1}[u]\right), \quad u^{\prime \prime}(0)=0, \quad u^{\prime}(1)=H_{2}\left(\alpha_{2}[u]\right), \quad u^{\prime \prime \prime}(1)=0,
$$

or

$$
u(0)=H_{1}\left(\alpha_{1}[u]\right), \quad u^{\prime \prime}(0)=0, \quad u^{\prime}(1)=0, \quad u^{\prime \prime \prime}(1)+H_{2}\left(\alpha_{2}[u]\right)=0,
$$

or

$$
u(0)=0, \quad u^{\prime \prime}(0)+H_{1}\left(\alpha_{1}[u]\right)=0, \quad u^{\prime}(1)=H_{2}\left(\alpha_{2}[u]\right), \quad u^{\prime \prime \prime}(1)=0,
$$

or

$$
u(0)=0, \quad u^{\prime \prime}(0)+H_{1}\left(\alpha_{1}[u]\right)=0, \quad u^{\prime}(1)=0, \quad u^{\prime \prime \prime}(1)+H_{2}\left(\alpha_{2}[u]\right)=0 .
$$

As in [12], where a different set of BCs were investigated, we point out that these nonlocal boundary conditions can be interpreted as feedback controls: for example, the BCs (4.1) can be seen as a control on the displacement at the left end and a device handling the shear force at $t=0$. 
Table 1

\begin{tabular}{ccccccc}
\hline & BCs (1.4)-(1.6) & BCs (4.1) & BCs (4.2) & BCs (4.3) & BCs (4.4) & BCs (4.5) \\
\hline$r_{1}(t)$ & $t$ & 1 & 1 & 1 & $-\frac{t^{2}}{2}+t$ & $-\frac{t^{2}}{2}+t$ \\
$\gamma_{2}(t)$ & $-\frac{t^{3}}{6}+\frac{t}{2}$ & $-\frac{t^{2}}{2}+t$ & $t$ & $-\frac{t^{3}}{6}+\frac{t}{2}$ & $t$ & $-\frac{t^{3}}{6}+\frac{t}{2}$ \\
$c_{\gamma_{1}}$ & $a$ & 1 & 1 & 1 & $2 a-a^{2}$ & $2 a-a^{2}$ \\
$c_{\gamma_{2}}$ & $-\frac{a^{3}}{2}+\frac{3 a}{2}$ & $2 a-a^{2}$ & $a$ & $-\frac{a^{3}}{2}+\frac{3 a}{2}$ & $a$ & $-\frac{a^{3}}{2}+\frac{3 a}{2}$ \\
\hline
\end{tabular}

Table 1 illustrates how the choice of the BCs affects the functions $\gamma_{1}, \gamma_{2}$ and the constants $c_{\gamma_{1}}, c_{\gamma_{2}}$.

Since one can see that

$$
1>2 a-a^{2}>-\frac{a^{3}}{2}+\frac{3 a}{2}>a, \quad a \in(0,1]
$$

the cone $K$, given by the constant $c$, varies according to the nonhomogeneous BCs considered. This affects also, in a natural manner, conditions (2.33) and (2.34).

\section{Acknowledgments}

The author would like to thank professor Salvatore Lopez of the Faculty of Engineering, University of Calabria, for shedding some light on the physical interpretation of this problem. The author wishes to thank the anonymous referees for their constructive comments.

\section{References}

[1] C. P. Gupta, "Existence and uniqueness theorems for the bending of an elastic beam equation," Applicable Analysis, vol. 26, no. 4, pp. 289-304, 1988.

[2] J. M. Davis, L. H. Erbe, and J. Henderson, "Multiplicity of positive solutions for higher order SturmLiouville problems," The Rocky Mountain Journal of Mathematics, vol. 31, no. 1, pp. 169-184, 2001.

[3] J. R. Graef and J. Henderson, "Double solutions of boundary value problems for $2 m$ th-order differential equations and difference equations," Computers $\mathcal{E}$ Mathematics with Applications, vol. 45, no. 6-9, pp. 873-885, 2003.

[4] Q. Yao, "An existence theorem for a nonlinear elastic beam equations with all order derivatives," Journal of Mathematical Study, vol. 38, no. 1, pp. 24-28, 2005.

[5] M. Eggesperger and N. Kosmatov, "Positive solutions of a fourth-order multi-point boundary value problem," Communications in Mathematical Analysis, vol. 6, no. 1, pp. 22-30, 2009.

[6] J. R. Graef, C. Qian, and B. Yang, "A three point boundary value problem for nonlinear fourth order differential equations," Journal of Mathematical Analysis and Applications, vol. 287, no. 1, pp. 217-233, 2003.

[7] J. R. Graef and B. Yang, "Positive solutions to a multi-point higher order boundary value problem," Journal of Mathematical Analysis and Applications, vol. 316, no. 2, pp. 409-421, 2006.

[8] J. Henderson and D. Ma, "Uniqueness of solutions for fourth-order nonlocal boundary value problems," Boundary Value Problems, vol. 2006, Article ID 23875, 12 pages, 2006.

[9] G. Infante and P. Pietramala, "A cantilever equation with nonlinear boundary conditions," Electronic Journal of Qualitative Theory of Differential Equations, no. 15, pp. 1-14, 2009. 
[10] B. K. Karna, E. R. Kaufmann, and J. Nobles, “Comparison of eigenvalues for a fourth-order fourpoint boundary value problem," Electronic Journal of Qualitative Theory of Differential Equations, no. 15, 9 pages, 2005.

[11] P. S. Kelevedjiev, P. K. Palamides, and N. I. Popivanov, "Another understanding of fourth-order fourpoint boundary-value problems," Electronic Journal of Differential Equations, no. 47, 15 pages, 2008.

[12] J. R. L. Webb and G. Infante, "Non-local boundary value problems of arbitrary order," Journal of the London Mathematical Society, vol. 79, no. 1, pp. 238-258, 2009.

[13] J. R. L. Webb, G. Infante, and D. Franco, "Positive solutions of nonlinear fourth-order boundary-value problems with local and non-local boundary conditions," Proceedings of the Royal Society of Edinburgh. Section A, vol. 138, no. 2, pp. 427-446, 2008.

[14] J. Yude and Y. Guo, "The existence of countably many positive solutions for nonlinear nth-order threepoint boundary value problems," Boundary Value Problems, vol. 2005, Article ID 572512, 18 pages, 2005.

[15] E. Alves, T. F. Ma, and M. L. Pelicer, "Monotone positive solutions for a fourth order equation with nonlinear boundary conditions," Nonlinear Analysis: Theory, Methods E Applications, vol. 71, no. 9, pp. 3834-3841, 2009.

[16] P. Amster and P. P. Cárdenas Alzate, "A shooting method for a nonlinear beam equation," Nonlinear Analysis: Theory, Methods E Applications, vol. 68, no. 7, pp. 2072-2078, 2008.

[17] A. Cabada and F. M. Minhós, "Fully nonlinear fourth-order equations with functional boundary conditions," Journal of Mathematical Analysis and Applications, vol. 340, no. 1, pp. 239-251, 2008.

[18] A. Cabada, R. L. Pouso, and F. M. Minhós, "Extremal solutions to fourth-order functional boundary value problems including multipoint conditions," Nonlinear Analysis: Real World Applications, vol. 10, no. 4, pp. 2157-2170, 2009.

[19] D. Franco, D. O’Regan, and J. Perán, "Fourth-order problems with nonlinear boundary conditions," Journal of Computational and Applied Mathematics, vol. 174, no. 2, pp. 315-327, 2005.

[20] F. Minhós, "Location results: an under used tool in higher order boundary value problems," in Proceedings of the International Conference on Boundary Value Problems, A. Cabada, E. Liz, and J. J. Nieto, Eds., vol. 1124 of Mathematical Models in Engineering, Biology, and Medicine, pp. 244-253, 2009.

[21] G. Infante, "Nonlocal boundary value problems with two nonlinear boundary conditions," Communications in Applied Analysis, vol. 12, no. 3, pp. 279-288, 2008.

[22] G. Infante and P. Pietramala, "Existence and multiplicity of non-negative solutions for systems of perturbed Hammerstein integral equations," Nonlinear Analysis: Theory, Methods \& Applications, vol. 71, no. 3-4, pp. 1301-1310, 2009.

[23] G. Infante and J. R. L. Webb, "Nonlinear non-local boundary-value problems and perturbed Hammerstein integral equations," Proceedings of the Edinburgh Mathematical Society. Series II, vol. 49, no. 3, pp. 637-656, 2006.

[24] G. L. Karakostas and P. Ch. Tsamatos, "Existence of multiple positive solutions for a nonlocal boundary value problem," Topological Methods in Nonlinear Analysis, vol. 19, no. 1, pp. 109-121, 2002.

[25] J. R. L. Webb and G. Infante, "Positive solutions of nonlocal boundary value problems: a unified approach," Journal of the London Mathematical Society. Second Series, vol. 74, no. 3, pp. 673-693, 2006.

[26] G. Infante, "Positive solutions of differential equations with nonlinear boundary conditions," Discrete and Continuous Dynamical Systems. Series A, supplement, pp. 432-438, 2003.

[27] G. Infante, "Nonzero solutions of second order problems subject to nonlinear BCs," in Dynamic Systems and Applications, Vol. 5, pp. 222-226, Dynamic, Atlanta, Ga, USA, 2008.

[28] K. Lan and J. R. L. Webb, "Positive solutions of semilinear differential equations with singularities," Journal of Differential Equations, vol. 148, no. 2, pp. 407-421, 1998.

[29] H. Amann, "Fixed point equations and nonlinear eigenvalue problems in ordered Banach spaces," SIAM Review, vol. 18, no. 4, pp. 620-709, 1976.

[30] D. J. Guo and V. Lakshmikantham, Nonlinear Problems in Abstract Cones, vol. 5 of Notes and Reports in Mathematics in Science and Engineering, Academic Press, Boston, Mass, USA, 1988.

[31] K. Q. Lan, "Multiple positive solutions of semilinear differential equations with singularities," Journal of the London Mathematical Society. Second Series, vol. 63, no. 3, pp. 690-704, 2001.

[32] J. R. L. Webb, “Optimal constants in a nonlocal boundary value problem," Nonlinear Analysis: Theory, Methods E Applications, vol. 63, no. 5-7, pp. 672-685, 2005. 